\title{
A novel technique for large and ptotic breast reconstruction using a latissimus dorsi myocutaneous flap set at the posterior aspect, combined with a silicone implant, following tissue expander surgery
}

\author{
Naohiro Ishii ${ }^{1}$, Jiro Ando ${ }^{2}$, Yusuke Shimizu ${ }^{3}$, Kazuo Kishi ${ }^{4}$ \\ ${ }^{1}$ Department of Plastic and Reconstructive Surgery, International University of Health and Welfare Hospital, Nasushiobara; ${ }^{2}$ Department of \\ Breast Surgery, Tochigi Cancer Center, Utsunomiya; ${ }^{3}$ Department of Plastic and Reconstructive Surgery, University of the Ryukyus Hospital, \\ Naha; ${ }^{4}$ Department of Plastic and Reconstructive Surgery, Keio University, Tokyo, Japan
}

\begin{abstract}
Large and ptotic breast reconstruction in patients who are not candidates for a transverse rectus abdominalis myocutaneous flap and revision surgery for the contralateral breast remains challenging. We developed a novel breast reconstruction technique using a latissimus dorsi myocutaneous (LD m-c) flap set at the posterior aspect of the reconstructed breast, combined with an anatomical silicone breast implant (SBI), following tissue expander surgery. We performed the proposed technique in four patients, in whom the weight of the resected tissue during mastectomy was $>500 \mathrm{~g}$ and the depth of the inframammary fold (IMF) was $>3 \mathrm{~cm}$. After over-expansion of the lower portion of the skin envelope by a tissue expander, the LD m-c flap was transferred to cover the lower portion of the breast defect and to achieve a ptotic contour, with the skin paddle set at the posterior aspect of the reconstructed breast. An SBI was then placed in the rest of the breast defect after setting the LD m-c flap. No major complications were observed during the follow-up period. The proposed technique resulted in symmetrical and aesthetically satisfactory breasts with deep IMFs, which allowed proper fitting of the brassiere, following large and ptotic breast reconstruction.
\end{abstract}

Keywords Surgery, plastic / Reconstructive surgical procedures / Tissue transplantation / Mastectomy / Breast

Received: 14 Dec 2017 • Revised: 11 Jul 2018 • Accepted: 13 Aug 2018

pISSN: 2234-6163 • elSSN: 2234-6171 • https://doi.org/10.5999/aps.2017.01781 • Arch Plast Surg 2018;45:484-489
Correspondence: Naohiro Ishii Department of Plastic and Reconstructive Surgery, International University of Health and Welfare Hospital, 537-3 Iguchi, Nasushiobara 329-2763, Japan

Tel: +81-287-39-3060

Fax: +81-287-39-3001

E-mail: ishinao0916@gmail.com

\section{INTRODUCTION}

In breast reconstruction, the shape of the reconstructed breast should be symmetrical with that of the contralateral breast to achieve a favorable aesthetic outcome. In addition, the inframa- mmary fold (IMF) should also be as symmetrical as possible to enable proper fitting of the brassiere. A transverse rectus abdominalis myocutaneous (TRAM) flap with revision surgery for the contralateral breast is the first treatment option for large and ptotic breast reconstruction. However, some patients with 
large and ptotic breasts are not candidates for a TRAM flap and revision surgery for the contralateral breast, and cannot achieve symmetrical breasts by implant-only breast reconstruction. A latissimus dorsi myocutaneous (LD m-c) flap and silicone breast implant (SBI) may be applied for breast reconstruction in such patients.

LD m-c flaps and SBIs have been mainly used for large breast reconstruction patients who have a skin infection due to an SBI or a skin disorder following radiotherapy, and for patients in whom the TRAM flap cannot be used [1-3]. In this method, the LD m-c flap is used to improve the upper thoracic morphology, the SBI is used to improve the lower thoracic morphology, and a skin island is exposed in the anterior view. However, it remains challenging to provide a symmetrical shape for the reconstructed breast, to create a deep IMF, and to avoid the exposure of a skin island.

Our study aimed to describe a novel technique to overcome these challenges, in which a LD m-c flap was used to improve the lower thoracic morphology, an SBI was used to improve the rest of the thoracic morphology after setting the LD m-c flap, and a skin paddle was placed at the posterior aspect of the re- constructed breast following tissue expander surgery.

\section{IDEA}

Between May 2016 and January 2017, we performed 2-stage breast reconstruction using an LD m-c flap and SBI following a simple mastectomy and immediate tissue expander insertion in four patients with breast cancer. This study included candidates for whom the weight of the resected tissue during mastectomy was $>500 \mathrm{~g}$ and the rostrocaudal distance along the chest wall between the lowest point of the breast and the IMF (the depth of the IMF) was $>3 \mathrm{~cm}$, and for those who did not want to receive breast reconstruction using a TRAM flap and revision surgery for the contralateral breast. None of the patients had a history of radiation therapy, and two patients had previously undergone postoperative adjuvant chemotherapy. The characteristics of all patients are shown in Table 1 .

We selected the expander (Allergan, Dublin, Ireland) according to the parameters of the contralateral breast and inserted the expander $2 \mathrm{~cm}$ below the lowest point of the contralateral breast to expand a skin envelope in the lower portion. We aimed to

\section{Table 1. Patient characteristics}

\begin{tabular}{|c|c|c|c|c|c|c|c|c|c|c|}
\hline \multirow[b]{2}{*}{ Case } & \multirow{2}{*}{$\begin{array}{l}\text { Age } \\
(\mathrm{yr})\end{array}$} & \multirow{2}{*}{$\begin{array}{c}\text { BMI } \\
\left(\mathrm{kg} / \mathrm{cm}^{2}\right)\end{array}$} & \multicolumn{3}{|c|}{ Breast parameter } & \multirow{2}{*}{$\begin{array}{l}\text { Regnault } \\
\text { classification } \\
\text { (grade) }\end{array}$} & \multirow{2}{*}{$\begin{array}{l}\text { IMF depth } \\
\text { (cm) }\end{array}$} & \multirow{2}{*}{$\begin{array}{l}\text { RW } \\
\text { (g) }\end{array}$} & \multirow{2}{*}{$\begin{array}{l}\text { Full saline } \\
\text { volume } \\
(\mathrm{mL})\end{array}$} & \multirow{2}{*}{$\begin{array}{c}\text { Follow-up } \\
\text { period } \\
\text { (mo) }\end{array}$} \\
\hline & & & $\begin{array}{l}\text { Height } \\
\text { (cm) }\end{array}$ & $\begin{array}{l}\text { Width } \\
\text { (cm) }\end{array}$ & $\begin{array}{l}\text { Projection } \\
\text { (cm) }\end{array}$ & & & & & \\
\hline 1 & 40 & 24.6 & 12.0 & 13.5 & 7.3 & 2 & 3.8 & 768 & 750 & 21 \\
\hline 2 & 41 & 27.5 & 15.5 & 15.5 & 4.6 & 3 & 4.5 & 620 & 830 & 17 \\
\hline 3 & 46 & 35.1 & 12.0 & 17.0 & 4.4 & 3 & 4.4 & 586 & 980 & 17 \\
\hline 4 & 57 & 30.6 & 12.5 & 15.5 & 5.8 & 2 & 4.1 & 611 & 820 & 13 \\
\hline
\end{tabular}

BMI, body mass index; IMF, inframammary fold; RW, weight of the resected tissue during mastectomy.

\section{Fig. 1. Preoperative view after full expansion}

(A) Case $1,(B)$ case 2.
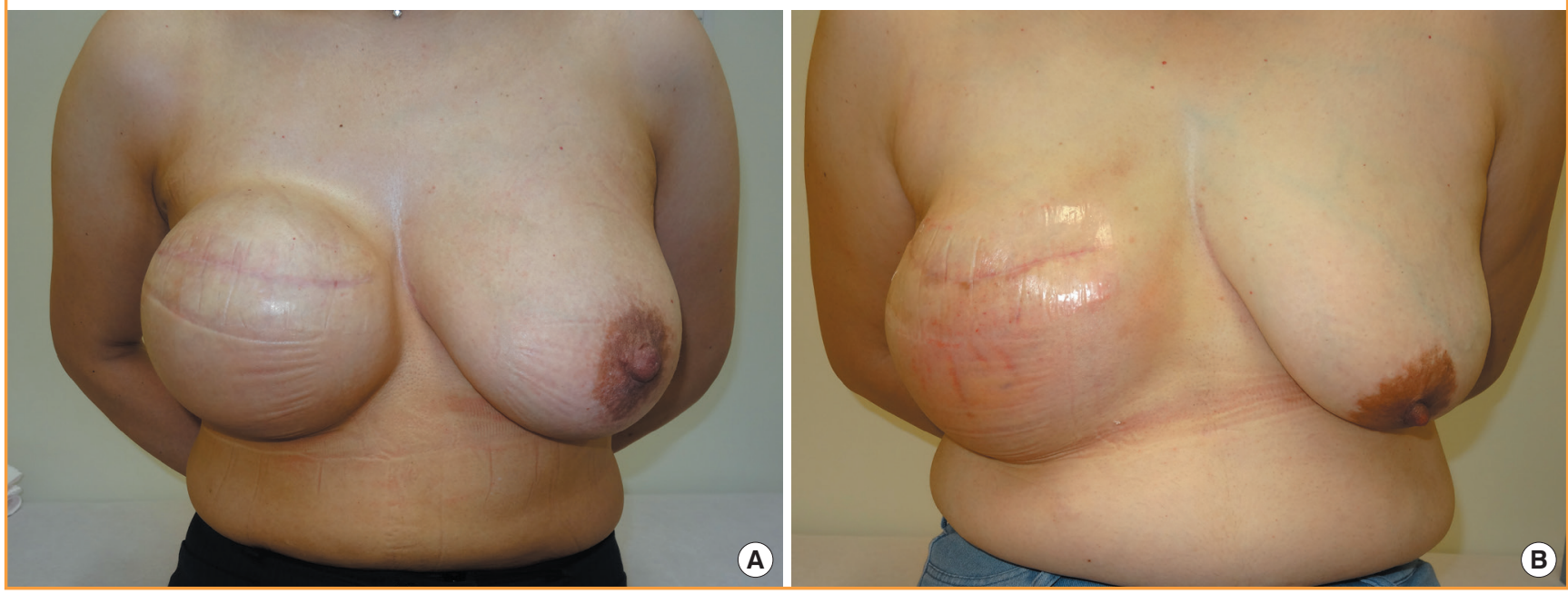


\section{Fig. 2. Transfer of the LD m-c flap}

A pedicled latissimus dorsi myocutaneous (LD $\mathrm{m}$-c) flap was transferred to the lower portion of the breast defect using an inframammary fold incision. (A) Case 1, (B) case 2.

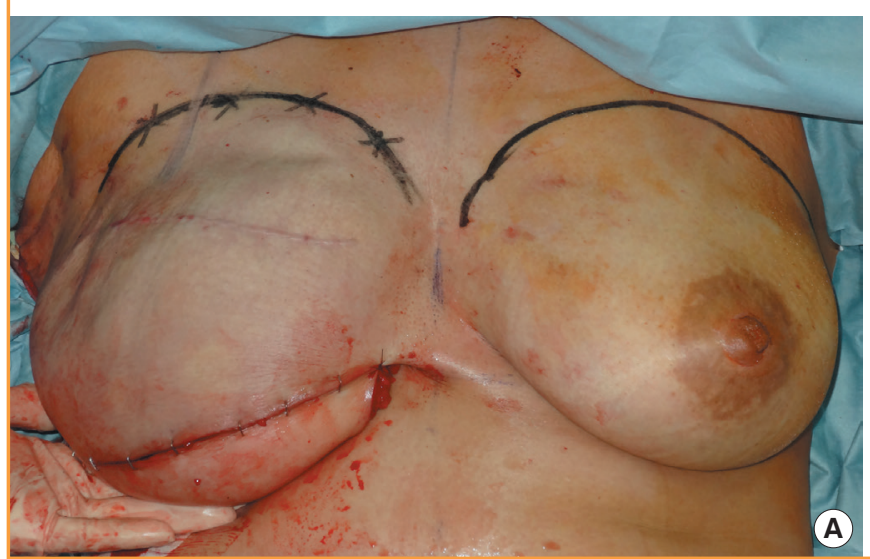

perform expansion until the skin envelope was approximately 1.3 times as large as the contralateral breast, which was chosen based on a consideration of shrinkage of the skin envelope according to the volume of saline in each expander and the degree of expansion of the skin envelope. Expander replacement was performed at least 3 months after saline injection was completed (Fig. 1).

The pedicled LD m-c flap was harvested with the skin paddle located obliquely, extending to the area superior to the iliac crest. The thoracodorsal nerve was preserved. Preoperatively, we estimated the LD weight according to the formula described in a previous report [4]. We then estimated the weight of the SBI (Allergan) from the estimated LD weight and the weight of the resected tissue during mastectomy. The weight of the LD $\mathrm{m}$-c flap decreases postoperatively because of its atrophy to less than the estimated LD weight; therefore, the sum of the estimated LD weight and the SBI weight should be more than the weight of the resected breast tissue. The dimensions of the SBI were selected according to the height and width of the contralateral breast measured with a ruler and the projection of the upper portion of the contralateral breast measured using ultrasound. Therefore, we prepared several kinds of SBIs, which were all of the anatomical type.

A skin incision was made at a site symmetrical to the IMF of the contralateral breast. The tissue expander was removed and the pedicled LD m-c flap was transferred to the lower portion of the breast defect, with the skin paddle placed at the posterior aspect of the reconstructed breast (Fig. 2). After setting the LD $\mathrm{m}$-c flap, the appropriate SBI was decided according to the dimensions of the rest of the breast defect and placed under the pectoralis major muscle without making contact with the distal flap to reduce the risk of postoperative infection due to the un-

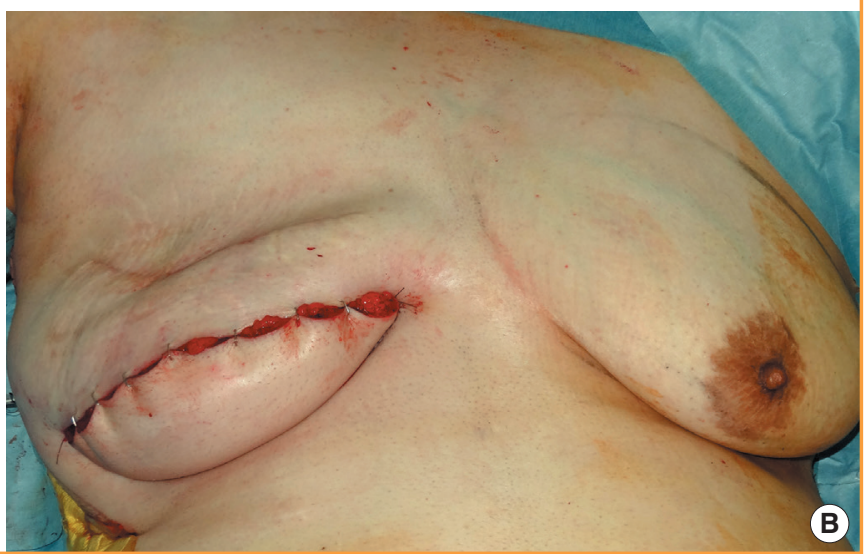

Fig. 3. Placement of the flap and implant

A silicone breast implant (SBI) was placed in the rest of the breast defect after setting the latissimus dorsi myocutaneous ( $L D$ m-c) flap under the pectoralis major muscle, above the LD. The LD m-c flap was rolled distally, without making contact with the SBI due to the unreliable circulation of the distal flap. PM, pectoralis major muscle.

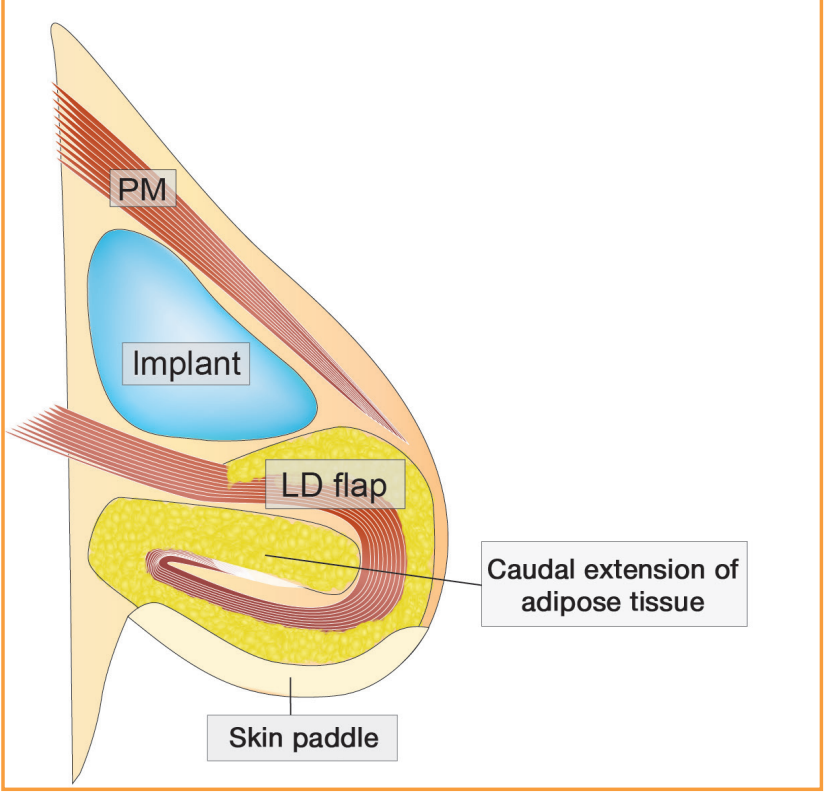

reliable circulation of the distal flap (Fig. 3). The flap volume can be controlled to reduce the size of the distal and rolled flap. We recommend choosing the flap volume based on a consideration of postoperative atrophy.

Touch-up surgery was performed at approximately 3 months after reconstruction with the LD m-c flap and SBI. We evaluated the postoperative results, which included aesthetic results and depth of the IMF 1 year after reconstruction with an LD m-c flap and SBI. Postoperative aesthetic results were evaluated ac- 


\section{Fig. 4. Postoperative view after reconstruction with proposed technique}

After reconstruction using the proposed technique, volume reduction of the flaps and nipple-areola plasty were performed. We successfully achieved symmetrical and satisfactory aesthetic results. (A) At 1 year and 6 months after reconstruction and 1 year and 3 months after touch-up surgery with the proposed technique in case 1. (B) At 1 year and 4 months after reconstruction and 1 year and 1 months after touch-up surgery with the proposed technique in case 2. (C) At 1 year and 6 months and 1 year and 3 months after touch-up surgery after reconstruction in case 1 , a symmetrical position of the bilateral inframammary fold was observed.
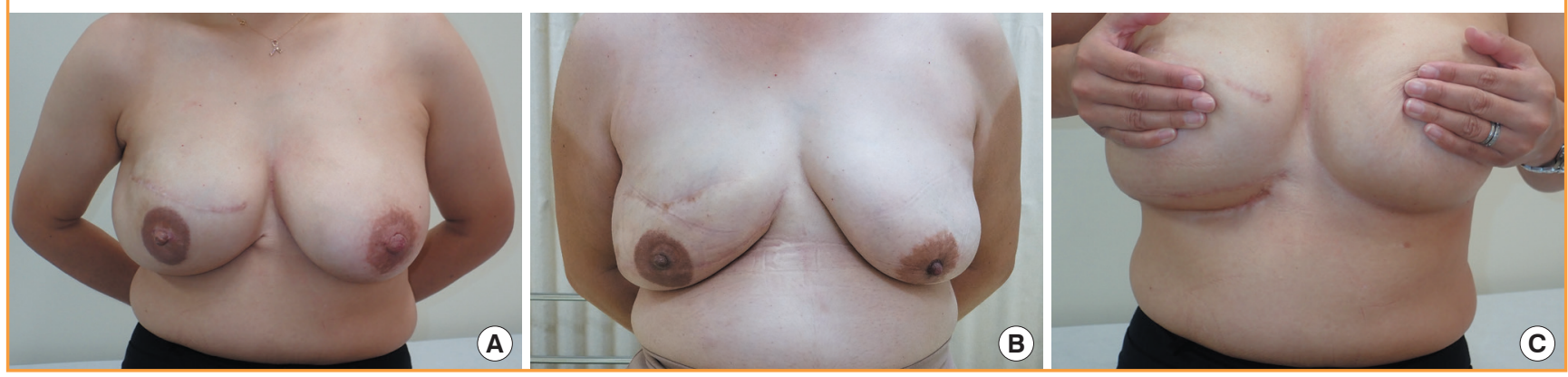

Table 2. Intraoperative and postoperative results

\begin{tabular}{|c|c|c|c|c|c|c|c|}
\hline Case & $\begin{array}{c}\text { Skin paddle } \\
\text { (height } \times \text { width, } \mathrm{cm} \text { ) }\end{array}$ & $\begin{array}{l}\text { Rostrocaudal LD flap } \\
\text { (height } \times \text { width, cm) }\end{array}$ & $\begin{array}{l}\text { Estimated LD } \\
\text { weight (g) }\end{array}$ & $\begin{array}{c}\text { SBI } \\
\text { weight }(g)\end{array}$ & $\begin{array}{c}\mathrm{SBI} \text { dimensions } \\
\text { (height } \times \text { width } \times \text { projection, } \mathrm{cm} \text { ) }\end{array}$ & $\begin{array}{l}\text { Resulting IMF } \\
\text { depth (cm) }\end{array}$ & $\begin{array}{l}\text { Cosmetic } \\
\text { result }\end{array}$ \\
\hline 1 & $7.5 \times 18.0$ & $13.5 \times 18.0$ & 316 & 550 & $13.2 \times 14.5 \times 6.7$ & 3.5 & Good \\
\hline 2 & $7.0 \times 19.0$ & $8.0 \times 19.0$ & 376 & 425 & $12.0 \times 14.5 \times 4.8$ & 4.7 & Good \\
\hline 3 & $6.5 \times 21.0$ & $10.0 \times 21.0$ & 534 & 270 & $10.5 \times 15.0 \times 3.6$ & 4.8 & Good \\
\hline 4 & $7.0 \times 20.0$ & $10.0 \times 20.0$ & 440 & 360 & $12.0 \times 14.5 \times 4.0$ & 4.2 & Good \\
\hline
\end{tabular}

cording to three grades (good, fair, and poor) jointly with each patient, considering patients' satisfaction.

The protocol for this study was approved by the relevant institutional review board (C-165), and all authors adhered to the Strengthening the Reporting of Observational Studies in Epidemiology (STROBE) guidelines. All patients provided written informed consent for the publication of this article and the use of their images.

During the follow-up period, there were no major complications, such as infection, flap necrosis, and SBI deviation, associated with reconstruction using the LD m-c flap and SBI. Touchup surgery under local anesthesia involved volume reduction of the flaps and nipple-areola plasty.

We achieved a symmetrical breast contour and IMF, allowing for proper fitting of the brassiere in all cases. Additionally, we achieved satisfactory aesthetic results since the skin paddle was not visible in the frontal view (Fig. 4). The estimated weight and size of the flaps, weight and dimensions of the SBI, and postoperative results are shown in Table 2.

\section{DISCUSSION}

Breast reconstruction with the LD m-c flap and SBI was first reported by Bostwick et al. [5] and has been used generally since.
In the 1970s, a radical mastectomy, including resection of the pectoral major and minor muscles, was usually performed; therefore, the LD or LD m-c flap was often used to provide safe coverage of the SBI. However, the development of the TRAM flap and tissue expanders have led to limitations in the application of the LD-mc flap and SBI; furthermore, mastectomy procedures became less invasive, allowing preservation of the pectoral muscle. Until now, studies regarding the use of the LD m-c flap and SBI in implant-based reconstruction have reported its use after radiotherapy or when a TRAM flap was unavailable $[1,2]$. In patients with less ptotic breasts, this method reduced exposure of a skin island by using a tissue expander [6]. Breast reconstruction with autologous tissue and artificial materials tends to be complicated; therefore, the LD m-c flap and SBI may not be the first treatment choice.

In breast reconstruction with a LD m-c flap and SBI for skin that is thin and scarred due to infection or radiotherapy, the flap is usually used to replace and reinforce a skin envelope, while the rest of the defect is covered by the SBI. In breast reconstruction with a LD m-c flap and SBI for a large breast not suitable for a TRAM flap, until now, the procedure has been as follows: a skin incision is made at the mastectomy scar; the flap then covers the upper thoracic morphology, and the SBI is used to improve the lower thoracic morphology. However, it is difficult to 
create a deep IMF and to avoid the exposure of a skin island. Furthermore, in an implant-only based reconstruction, even if a tissue expander is used, there are limitations to the depth of the IMF that can be created [7].

The proposed technique enables the creation of a deep IMF with the skin paddle of the LD m-c flap set at the posterior aspect of the reconstructed breast and a high projection at the inferior site using both the LD m-c flap and an SBI. In addition, it uses an expanded skin envelope in the lower portion of the breast to achieve a symmetrical, ptotic breast while controlling the flap volume. Furthermore, an invisible skin paddle in the frontal view results in highly favorable aesthetic outcomes. In recent breast reconstructions, many patients desire a less invasive method; however, some are not candidates for a TRAM flap and revision surgery for the contralateral breast even though they have large and ptotic breasts. For such patients, the proposed technique can be used to achieve satisfactory results with minimal complications.

Over-expansion should be performed to create a skin envelope of sufficient size for patients with highly ptotic breasts. The peak of projection in a large and ptotic breast is located at the inferior site, and the height of the rest of the breast defect after setting the LD m-c flap is less than its width in many cases; therefore, an anatomical implant was generally selected rather than a round implant. To avoid a bi-lobed appearance with the SBI and the LD m-c flap, we overwrapped the LD m-c flap with the lower portion of the SBI. It may be possible to avoid a bi-lobed appearance in patients with a thick skin envelope.

In the literature, $5 \%$ to $50 \%$ of patients required further surgery for exchange or temporary removal of an SBI, and furthermore, $10 \%$ of patients needed definitive removal of the SBI at a later date [8-10]. Photographic assessment of the long-term results showed that the objective aesthetic results were insufficient in a considerable number of cases [9]. The reconstructed breast may evolve over time differently from the contralateral breast, making predictions challenging, which can cause it to be difficult to achieve long-term symmetry [10]. The SBI was placed above the LD m-c flap in the present cases; thus, it was not expected to be influenced by postoperative contracture of the $\mathrm{LD}$ $\mathrm{m}$-c flap. Many complications in breast reconstruction using the LD m-c flap and SBI have been reported, as described above, and the technique proposed in the present study has not been reported to date; therefore, larger case studies of the proposed technique with careful long-term follow-up are needed. The patients in this study provided sufficient informed consent, including the possibilities of postoperative revision; SBI dislocation, collapse, and infection; and unexpected complications.

The weight of breasts that can be reconstructed with the pro- posed technique should be considered. The maximum weight of an applicable SBI in general clinical experience is about $600 \mathrm{~g}$, and the weight of a harvestable LD m-c flap ranges from 100 to $400 \mathrm{~g}[4,11]$. Therefore, patients with a contralateral breast weight of $<800 \mathrm{~g}$ are eligible for this technique considering the postoperative atrophy of the flap. Furthermore, it may be difficult to apply this technique in patients with a very high projection or a peak of projection located in the most inferior area in the contralateral breast.

In conclusion, although further research may be required, including studies with a larger sample size and careful long-term follow-up, the present study showed that this technique may be useful in patients with large and ptotic breasts who are not candidates for a TRAM flap. The LD m-c flap technique could overcome limitations of the treatments that are currently in wide use.

\section{NOTES}

\section{Conflict of interest}

No potential conflict of interest relevant to this article was reported.

\section{Ethical approval}

The study was approved by the Institutional Review Board of Tochigi Cancer Center (IRB No. C-165) and performed in accordance with the principles of the Declaration of Helsinki. Written informed consents were obtained.

\section{Patient consent}

The patients provided written informed consent for the publication and the use of their images.

\section{REFERENCES}

1. Leff DR, Bottle A, Mayer E, et al. Trends in immediate postmastectomy breast reconstruction in the United Kingdom. Plast Reconstr Surg Glob Open 2015;3:e507.

2. van Huizum MA, Hage JJ, Rutgers EJ, et al. Immediate breast reconstruction with a myocutaneous latissimus dorsi flap and implant following skin-sparing salvage mastectomy after irradiation as part of breast-conserving therapy. J Plast Reconstr Aesthet Surg 2016;69:1080-6.

3. Giacalone PL, Bricout N, Dantas MJ, et al. Achieving symmetry in unilateral breast reconstruction: 17 years experience with 683 patients. Aesthetic Plast Surg 2002;26:299302.

4. Kubo T, Miki A, Osaki Y, et al. Simple volume estimation of 
the latissimus dorsi musculocutaneous flap in Asian breast reconstruction. J Plast Surg Hand Surg 2014;48:148-51.

5. Bostwick J 3rd, Vasconez LO, Jurkiewicz MJ. Breast reconstruction after a radical mastectomy. Plast Reconstr Surg 1978;61:682-93.

6. Feng J, Pardoe CI, Mota AM, et al. Two-stage latissimus dorsi flap with implant for unilateral breast reconstruction: getting the size right. Arch Plast Surg 2016;43:197-203.

7. Ishii N, Ando J, Harao M, et al. A new criterion for the application of 2-stage implant-only breast reconstruction using a classification based on the rostrocaudal distance along the chest wall between the lowest point of the breast and inframammary fold. Eplasty 2017;17:e23.
8. De Mey A, Lejour M, Declety A, et al. Late results and current indications of latissimus dorsi breast reconstructions. Br J Plast Surg 1991;44:1-4.

9. Tarantino I, Banic A, Fischer T. Evaluation of late results in breast reconstruction by latissimus dorsi flap and prosthesis implantation. Plast Reconstr Surg 2006; 117:1387-94.

10. Berdah-Benjoar Y, Masson J, Revol M, et al. Late results in breast reconstruction by latissimus dorsi flap and prothesis implantation. Ann Chir Plast Esthet 2009;54:295-302.

11. Fujiwara T, Yano K, Tanji Y, et al. Preoperative estimation of pedicled latissimus dorsi flap weight for breast reconstruction. J Plast Reconstr Aesthet Surg 2014;67:579-81. 